\title{
An Approach to the Cooperative Management of Multitechnology Networks
}

\author{
Raquel Díaz-Caldera and Juan Serrat-Fernández, Universitat Politècnica de Catalunya \\ Klearchos Berdekas and Fotis Karayannis, National Technical University of Athens
}

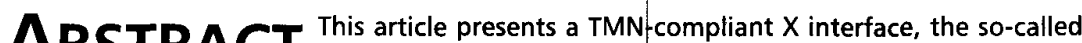
$X$ coop interface, as a fundamental component of an operations system developed to cope with the challenges of multidomain, multitechnology networks. Defined at the network level, between operations systems of different TMN domains, it is intended as a support for the management of broadband connectivity services with endto-end QOS and scheduling constraints in backbones operated by many network operators. The key feature of the present approach, distinguishing it from other existing Xcoop solutions, is in handling the integrated ATM and SDH technologies, covering both the Configuration Management and Fault Management functional areas.
\end{abstract}

Teday's globalization of the economy sets new requirements for worldwide operators, since multinational companies demand, and gradually depend on, end-to-end broadband connections spanning many operators' domains. In the emerging deregulated multitechnology, and multiprovider telecommunications world puzzle, the management of such end-to-end connections reaches high complexity levels, requiring operators to be both cooperators and competitors to serve the many customers' needs.

An intuitive approach to dealing with this task is to rely on service-level management. Nevertheless, the requirements of modern services based on dynamic tariffs and bandwidth brokerage tend to extend far beyond the functions typically assigned to service-level operations systems. In such an encompassing scenario, the provision of such services should rather be based on the management of raw bandwidth, inside a global multidomain backbone operated by many network operators. At the network level, enabling the selection between technologies - asynchronous transfer mode (ATM), native synchronous digital hierarchy (SDH), or native wavelengthdivision multiplexing (WDM) - for establishing a connection between peer points in different domains is reasonable. Therefore, the availability of an integrated multitechnology management service can be perceived as the ideal solution.

Cooperation between management systems is allowed if they have properly defined interfaces. In the telecommunications management network (TMN) framework [1], thes interfaces are X-type interfaces, called Xcoop (cooperative) interfaces due to the specific purpose for which they are intended.

The specification and implementation of Xcoop interfaces have attracted much attention in the last few years. In fact, the appropriate design of this system component is a key aspect of efficient and seamless cooperative management. In this context, it is worth mentioning the EURESCOM P408 project [2], which produced the specification of an Xcoop interface for ATM-based networks and a different one for SDH-based networks. The European Telecommunication Standards Institute (ETSI) has issued two standards related to the configuration management and fault management of ATM networks [3]. Recently, ETSI launched the EASI project [4] which, in collaboration with EURESCOM, is contributing to the identification of interoperability requirements for the production of related specifications in the context of ATM multidomain networks. In addition, the ATM and Telemanagement Fora have working groups and specifications on the topic, although the latter is addressed more toward pure service-level models [5].

The Xcoop specification presented in this article is a step ahead in the evolutionary process of this system interface. Distinguishing it from previous work, it permits interactions between management systems independent of the underlying network technology - ATM, SDH, or hybrid. This is achieved by defining the appropriate functionality and presenting an information model, where, in fact, the specific characteristics of the ATM and SDH resources are abstracted and merged in common classes.

The work presented here was undertaken as part of the EU ACTS project MISA [6] and is focused on the requirements, specification, and implementation of an Xcoop interface for ATM and SDH networks. The next section presents an overview of topics to cover in the context of multidomain management. In the following one, the MISA approach is presented as a solution for the ongoing challenges. Finally, the last two sections are devoted to the Xcoop interface itself and also include a scenario involving the key MISA components in a representative network environment.

\section{ISSUES IN MULTIDOMAIN NETWORK MANAGEMENT}

By selecting a business case rather than a technical one, we consider how a value-added service provider (VASP) can offer one-stop shopping for videoconferencing services. These services could be directed to international corporations for which videoconference services can provide advantages, such as security companies, hospitals, manufacturers offering remote repair services, educational institutions, and entertainment enterprises. To provide these videoconferencing services, the VASP should use bearer connectivity services provided by public network operators (PNOs). From the VASP point of view the services should exhibit, among other properties, seamless international coverage, broadband connectivity, and easy access through defined service access points (SAPs). These SAPs should fulfill the VASP users' needs, allowing them the service specification in terms of quality of service (QoS), bandwidth, scheduling, and other requirements. In the context of this article, these types of services are referred to as broadband connectivity services (BCS).

The above commercial example is just one among many 
that give an example for highlighting the different applications of these BCSs. Of course, each application may require different service profiles.

The potential user of a BCS will then subscribe to at least one PNO. Therefore, PNOs will face a situation characterized by a diversity of elements, including different types of requests (QoS, scheduling) to be satisfied making use of networks belonging to other PNOs, which often have different network technologies as well.

This proposed solution defines a BCS provided through a network management service with several management functional areas at different operational levels. The basic user need is the establishment and use of connections; therefore, the first requirement for the management service is to provide Path Provisioning functionality. Moreover, Fault Management also emerges as a basic functionality, to minimize service outage and user dissatisfaction. While fault management operations should, in general, be transparent to the user, his or her right to be kept informed of the connection status should be respected (e.g., during a fault recovery process), and should be provided directly by the Fault Management service. Security and Accounting Management also play fundamental roles in a multidomain context. Each involved PNO should offer resources to users (other PNOs) through open interfaces in order to set up multidomain connections. This entails the risk that nonauthorized users may also have access. Billing is inherent to the Path Provisioning service and is the object of Accounting Management. It is clear that for multidomain connections, the accounting processes will require billing information transfers through domains, as for path provisioning and fault management. Configuration and fault management specification and implementation are described in this work; accounting management is under specification, while security management remains for future research.

\section{Configuration Management Requirements}

Users' needs dictate many requirements on the path provisioning service, referred to hereafter. This service should provide end-to-end connectivity across networks belonging to different management domains that have a connection segment in each domain. Moreover, these networks may use different transmission technologies. To serve all business cases, there is a need to provide the establishment, modification, release and reconfiguration of connections, taking care of the requested QoS, bandwidth, and scheduling.

In a generic context, one can assume that once a user has subscribed to a domain, he or she can request a connection that will be initiated by this domain, termed the originating domain. The role of the originating domain may be different later, depending on the adopted organizational model. Two classical approaches are candidates here, namely star and cascade.

In the star organizational model, the originating domain cooperates directly with all other associated domains to establish, maintain, and terminate the segments of the end-to-end connection. This model is quite rigid and only appropriate in networks with a few nodes, keeping in mind that the organizational model also has implications in all other management functional areas. For example, in the star model the alarms related to any connection segment are sent to the originating domain because it is the unique domain, which keeps track of the entire end-to-end connection. The advantage of this model is that it has fewer states, and is therefore simpler to implement.

In a pure cascade organizational approach each domain is responsible for the next segment of the end-to-end connection. This model is especially powerful when used in combination with the star model, forming a hybrid model. This hybrid approach is the most flexible because it allows the definition of many hierar- chical levels that simplify system scalability, although the most complex due to different allowable interdomain relationships.

A key issue in dealing with path provisioning is routing. The BCS must be based on QoS, and schedule constrained routing algorithms to match user needs and network ressurces availability at a given time. Regardless of the principles or effectiveness of one or another routing algorithm, each PNO must be allowed complete freedom of choice. In fact, routing may entail not only technical but also policy aspects. 'Therefore, the BCS service must meet this requirement.

Reservation, activation, and deactivation must be included in establishing the connection. The key aspect in the reservation process is the information that should be provided (QoS, bandwidth, schedule, etc.). The alternative to specifying all detailed requirements in the request consists of specifying only thə identifier of a connection whose characteristics are known in advance, by means of a proactive advertising mechanisin from the remote domains (all domains advertise their potential connections characteristics to all domains). This advertising multicast of connection characteristics can be viewed as either a mechanism to minimize the management traffic load on the data communication network or a business process enabling a PNO to compete against the offers of other PNOs.

Activation, deactivation, and release of connections ave in common the fact that they point only to a connection identifier, already defined at reservation time; their functionality is not considered especially demanding in a cooperative management context.

\section{Fault Management Requirements}

Users' requirements can also be mapped into the Fault Management service. To enhance resilience of connections, this service should provide the recovery of connections affected by faults in the minimum possible time, informing the users of the BCS about the most relevant fault correction actions in jrocess (if any), and logging of the events. The management sysiems of different domains will therefore need to exchange fault management information across domain boundaries in order to support, at minimum, the reporting of alarms, processing of alarms, reporting of recovery actions, and logging of alarm everits.

The need to exchange fault management information across domains can be a problem because this type o: information may be seen by the PNOs as highly sensitive, and therefore not to be disclosed to competitors. This problem, however, can be solved if design of the BCS service is driven by appropriate guidelines. First of all, a clean and powerful information model must be supported, and its entitie:s must completely hide (abstract) the real network resources that are represented. In this way, the entity "connection," say for PNO-A, may be just an ATM virtual path between two ATM switches, while for PNO-B it may be a complex link involving different subnetwork connections based on ATM and SDH. In this way, the abstract object "connection"sends arı alarm notification out of its domain, advising that something is wrong in the "connection," but without giving anything, about the real nature of the failure. Another design princip e must be to leave each domain to carry out as much alarm processing and recovery processes as possible, in order to minimize interaction with other domains.

Alarm correlation is also considered mandatory in the BCS service for two reasons. Since the BCS service provides an integrated management of both ATM and SDH technologies, the correlation among alarms issued by the technology-defendent management systems is necessary. As an example, in the case of a domain with hybrid ATM/SDH supported connections and a failure occurring in the SDH subnetwork, not only the S.JH but also the ATM subnetwork management system will receive 
and issue alarms. The alarms coming out from the ATM subnetwork are secondary, and this will be discovered during the correlation of all the alarms emitted by the two sources, where the SDH loss-of-signal alarm will be identified as the primary alarm. The primary alarm is associated with the faulty resource and provides the BCS service with enough information to trigger a recovery process. In this example, the correlation occurs among alarms produced inside one domain, with a further assessment correlating alarms from the different domains.

\section{The Solution Provided by the MISA PROJECT}

One of the goals of the MISA project [6] has been to develop a global broadband connection (GBC) service, supporting both ATM and SDH networks, belonging to the same or different network operators. The MISA proposal provides users and network operators with a management service of connections by means of a system defined in the TMN framework. This global system consists of operation systems (OSs), one in each PNO domain, which in turn are supported by other low-level OSs.

The MISA system, then, is a solution to the issues just raised in the previous sections. What was called the BCS service shall be now called the GBC service. Starting with an overview of the MISA concepts, let us say that MISA is a TMN-based approach. Other approaches - based on Telecommunications Information Network Architecture (TINA) or Common Object Request Broker Architecture (CQRBA), for instance - could have been used as well. The reasons to move to the TMN direction have been first to examine the feasibility of the TMN approach in a multidomain environment, as well as management systems around the world, at the same time allowing the setup of a real international trial environment. to exploit the already existing investment in deployed network

Figure 1 shows the architecture of the MISA OS and its level in the TMN pyramid. The lowest level of the MISA OS is the integrated network level, which deals with the integration aspects of the two network technologies, ATM and SDH, in each domain; this level functions to maintain and terminate connections inside the local domain. Over the integrated network level is the cooperative network level, which is activated when the connection should be supported by more than one domain. At the apex of the three-level architecture of Fig. 1 is the service level, which supports the interaction of the user with the GBC system.

Looking at the MISA OS architecture, routing appears divided into two levels: interdomain and intradomain. Interdomain routing will be entrusted to split the end-to-end connection into connection segments that have a one-to-one relationship between connection segments and PNOs assigned. This splitting may be based on geographical constraints, of course, but may also be based on global service agreements between the competing PNOs, or on more dynamic resource allocation criteria. This routing level is a feature of the GBC service. On the other hand, the intradomain-level routing must be completely transparent because it is a feature of each intermediate PNO within its own domain. A more detailed description of the routing systems considered in MISA can be found in [7]. A similar situation exists for the alarm correlation process, where intradomain correlation takes place in alarm correlator 2 , whereas interdomain correlation is undertaken in alarm correlator 1 . The highest priority is assigned to the intradomain level to solve any failure affecting resources inside the domain, providing external indication of a problem that will be resolved without external intervention. Only when the problem cannot be resolved inside the domain will it allow an external system to bypass the faulty resource, but, even in this case, without giving further information.

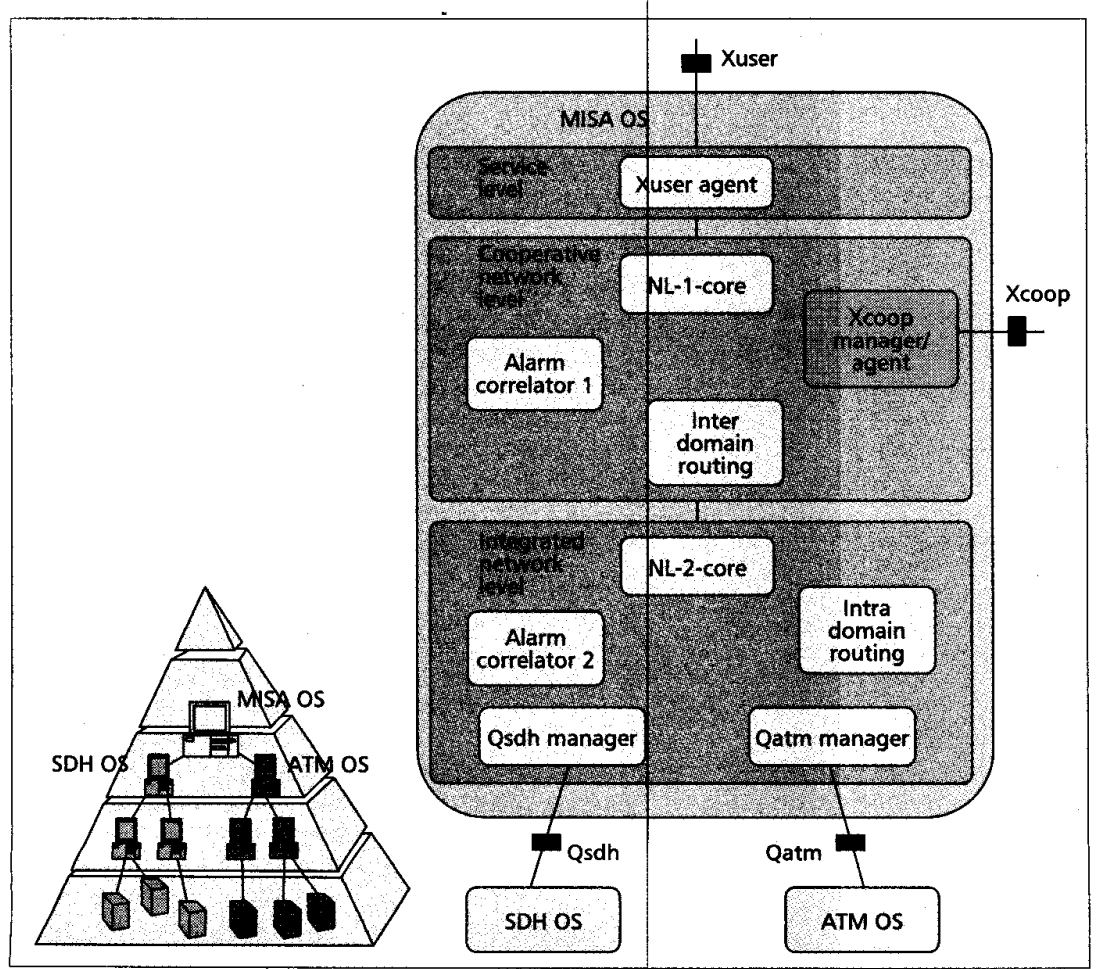

Figure 1. The MISA OS architecture.
The NL1-Core and NL2-Core modules are key elements for which the behavior of the corresponding level has been programmed. The information reaching one of the two network levels is first analyzed by the corresponding core and then may either process this information, start one of the modules of the same level, or send out information to the other core.

The MISA OS interacts with the environment through the four external interfaces shown in Fig. 1. The Xuser is an X-type interface through which the user only sees a seamless network, although it may span different domains, and may be based on different technologies. With this interface, the user can subscribe to any SAPs, and/or request connections between SAPs, and be notified of the progress of these requests or about any abnormal behavior of any existing connection. On the right can be seen the Xcoop, also an X-type interface, for the exchange of information between different domains. The following sections provide more details about the interface. On the bottom of Fig. 1 are two different Q-type interfaces intended to allow the interaction of the MISA OS with technology-dependent OSs. 
These two Q-type interfaces were also specified for the MISA project. In order to carry out field trials during project execution, it was necessary to adapt the existing ATM OSs and SDH OSs to the information models of the Q-type interfaces. The adaptation was site-dependent, and is not shown explicitly in the figure. These interfaces conform to the manager/agent paradigm, and the managers and agents represented in Fig. 1 are solely for their support.

\section{Functional ARChitecture of the XCOOP INTERFACE}

The specification of the Xcoop model interface in MISA was undertaken in compliance with the methodology defined in the Network Management Forum (NMF) Ensembles [8]. In this context, the functional architecture is specified in terms of managed resources, management function sets, and management functions. For readability the resources are briefly described, and functions are summarized in Tables 1 and 2; the use of these functions in a conventional scenario is also illustrated

Externally, the MISA OS reveals an abstracted view of its physical network through the Xcoop interface. A total of four such abstract objects/managed resources have been defined, regardless of whether the underlying networks are pure ATM, pure SDH, or hybrid ATM over SDH.

The most generic resource is the GBC Subnetwork that abstracts the entire operator network; the GBC Subnetwork Access Point is distinguished at the network boundaries. This resource abstracts either the port of an ATM access switch or a termination point of an SDH multiplexer. At the same time, any of the access points can be the endpoint of a link between the local and a remote domain, as well as the endpoint of many internal connections that cross the domain. The links between domains are abstracted by the resource called the GBC Inter-PNO Link, and a connection crossing the (local) domain is representeD by the GBC Subnetwork Connection.

The functionality of the Xcoop model covers four management function sets intended for Configuration Management and another four sets for Fault Management. In turn, each set

\begin{tabular}{|l|l|}
\hline Managenent Funguion Set & Management Function \\
\hline Alarms and Recovery & Alarm Reception \\
\hline Actions Processing & $\begin{array}{l}\text { Alarm Filtering and Correlation } \\
\text { Recovery Action Notification Reception } \\
\text { Recovery Action Triggering }\end{array}$ \\
\hline Alarm Reporting & $\begin{array}{l}\text { Report GBC Alarm to Destination User } \\
\text { Report Alarm from GBC Subnetwork Access Point } \\
\text { Report Alarm from GBC Subnetwork Connection } \\
\text { Report QOS Degradation }\end{array}$ \\
\hline Recovery Process Events \\
Reporting & $\begin{array}{l}\text { Report GBC Recovery Result to Destination User } \\
\text { Notification of Protection Switching on a } \\
\text { GBC-Inter-PNO Link } \\
\text { Notification of Recovery Process Result on a } \\
\text { GBC-Subnetwork Connection }\end{array}$ \\
\hline Alarm Event Logging & $\begin{array}{l}\text { Request Aalarm Report } \\
\text { Alarm Events Logging } \\
\text { Alarm Events Reading }\end{array}$ \\
\hline
\end{tabular}

Table 2. Xcoop interface: Fault Management supported functionality.

\begin{tabular}{|l|l|}
\hline Wonf & Management Function \\
\hline Establish & $\begin{array}{l}\text { Destination User Checking } \\
\text { Get Available Domain Connections } \\
\text { Update Topology Information } \\
\text { Reserve GBC Subnetwork Connecticin } \\
\text { Activate GBC Subnetwork Connection } \\
\text { Notify End User }\end{array}$ \\
\hline Release & $\begin{array}{l}\text { Release GBC Subnetwork Connecticn } \\
\text { Deactivate GBC Subnetwork Connestion } \\
\text { Notify End User }\end{array}$ \\
\hline Modification & $\begin{array}{l}\text { Change GBC Subnetwork Connection } \\
\text { Activate GBC Subnetwork Connection } \\
\text { Notify End User }\end{array}$ \\
\hline Reconfiguration & $\begin{array}{l}\text { Reserve GBC Subnetwork Connection } \\
\text { Activate GBC Subnetwork Connection } \\
\text { Release GBC Subnetwork Connection } \\
\text { Deactivate GBC Subnetwork Connection } \\
\text { Notify End User }\end{array}$ \\
\hline
\end{tabular}

Table 1. Xcoop interface: Path Provisioning supported functionality.

includes several management functions. This structure is presented in Tables 1 and 2 for configuration and fault management, respectively. The mnemonics used are quite suggestive of the specific use of each set or function. An individualized description is beyond the scope of this article; the reader interested in such a description is referred to [9].

\section{Combined Use of SOME Functional COMPONENTS IN A SAMPLE SCENARIO}

Using the above function sets, the Xcoop interface can support many complex information exchange situations. Highlighting these possibilities, a scenario where the Xcoop s used to support the cooperative management of a connection based on hybrid ATM/SDH networks is described. Furthermo*e, this scenario presents the functional use of Xcoop as a module of the MISA OSs. This scenario thereby elaborates on the previous description of the MISA system.

Figure 2 presents the scenario in which, for simplicity, only two domains are considered - domains $\mathrm{W}$ and $\mathrm{E}$ - the first including a pure ATM network, the second a hybrid ATM/SDH network. These networks are managed by the appropriate OSs, as shown at the top of the figure with the interfaces indicated, while at the bottom of the figure the infrastructures are depicted as they are (abstractly) riewed at the Qatm or Qsdh interfaces. At the Qatm and Qsdh interfaces, and similar to Xcoop, managed resources have been defined as well. For instance, in ATM Subnetwork W, we have two access points (QatmW1 and Q $\overline{\text { atmW2), which }}$ are mapped to two Xcoop access points (GBC Subnetwork Access Points). On the side of domain E, the SDH_Subnetwork is a core network not directly accessible from the outside. None of the access points of this subne twork, therefore, is mapped to Xcoop access froints. Neither QatmE2 nor QatmE3 is seen at the Xcoop level, since they are considered intertechnology access points (between ATM and SDH networks), not interdomain points.

Consider that a connection request be tween QatmW1 and QatmE4 is received at MISA OS W. The connection will be successfully established, also involving the SDH_Subnetwork, as shown in Fig. 2. 
Subsequently, a fault will occur in the intertechnology link between QatmE2 and QsdhE1.

The time diagram in Fig. 3 is used to describe the system behavior. On receiving a connection request, the interdomain routing algorithm in MISA OS W determines that, in this case, the best route involves only the originating domain (domain $\mathrm{W}$ ) and the destination domain (domain $\mathrm{E}$ ); no transit domains exist. The end-to-end QoS parameters (QOSall) have already been "split" among the two domains (QOSw, QOSe); each domain is requested to meet such QoS restrictions which, when aggregated, will meet the end-to-end (user) requirements. An ATM connection request is sent to the local ATM OS W through the Qatm Manager (local domain), and also a reservation request is sent to the destination domain through the Xcoop interface (remote domain). This last request specifies the QoS (QOSe) and the endpoints of the connection segment (QatmE1, QatmE4), making use of the function Reserve GBC Subnetwork Connection. When received by MISA OS $E$, this request triggers the intradomain routing algorithm, which decides that the optimum route is through the SDH Subnetwork (as shown in Fig. 2). This triggers the three reservation requests shown on the right of Fig. 3 (for connections QatmE1-QatmE2, QsdhE1-QsdhE2, QatmE3-QatmE4). It is assumed that all these requests are positively confirmed. In addition, note that since the different requests and replies are asynchronous, they may occur in a sequence different from that shown in the figure.

When the three reservations in domain $\mathrm{E}$ are successfully completed and confirmed, the MISA OS of the destination domain sends the Reserve GBC Subnetwork Connection reply to the origin; thus, the originating MISA OS W is aware of the fact that the end-to-end GBC multidomain connection is reserved, hence informing both end users. The destination end user is informed through the Xcoop again, using the Notify End User function, completing the reservation functionality. The transactional character of the procedure should be noted; every step must be completed; if any step fails, the system has the mechanism to roll back, including the appropriate

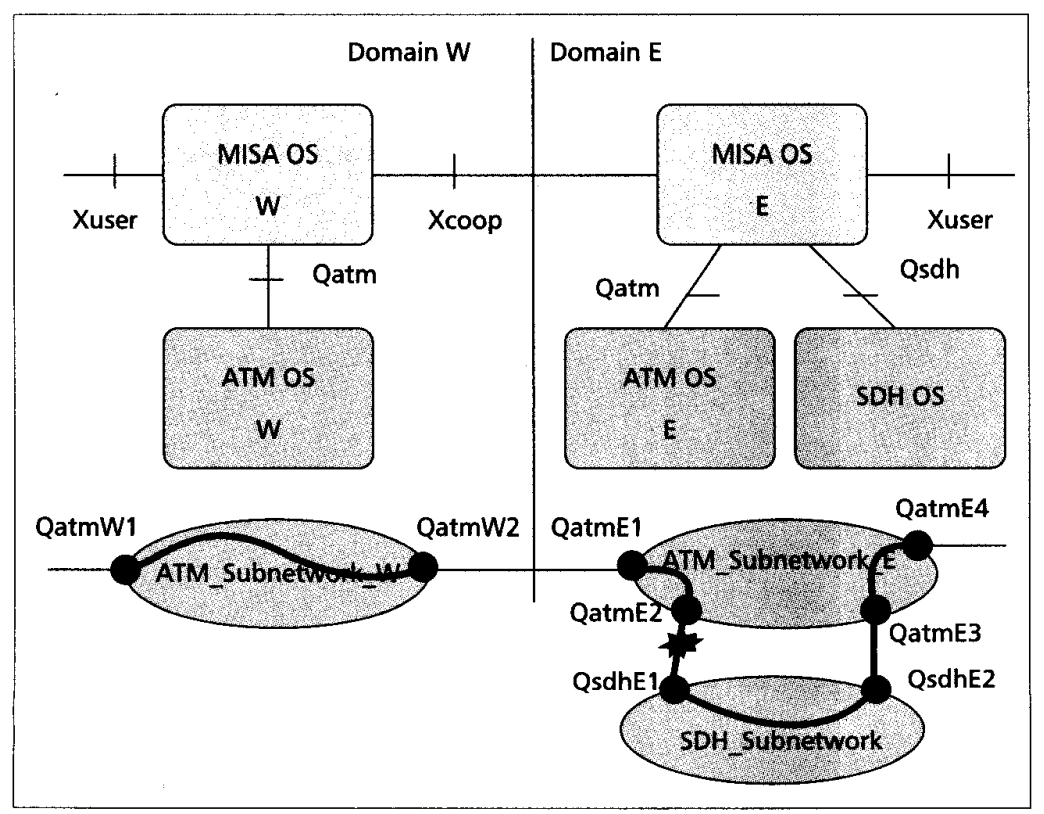

Figure 2. GBC connection in a multidomain, multitechnology scenario. release requests clearing any reservations "hanging out." The transactional principle of operations holds for all procedures defined in the Xcoop model.

When the end-to-end connection is reserved and, at a time afterward, either activated or deactivated, we assume a failure (e.g., the break of the intertechnology link shown in the figures). If the line break affects only the upward flow from the SDH toward the ATM subnetwork (cut one of the pair of fibers), the fault is detected as LOS at the QatmE2 ATM access point. The propagation of the operation, administration, and maintenance (OAM) cells and bytes at the ATM and SDH levels, respectively, trigger the emission of several alarm indication signal (AIS) and remote defect indication (RDI) alarms. All these alarms are represented by a pair of arrows inside a circle in Fig. 3.

The alarms arriving at the MISA OS E are all correlated in alarm correlator 2 , the intradomain alarm correlator module. At the completion of this correlation process, MISA OS E determines the cause of the alarm storm and triggers a local recovery procedure, while sending an alarm (and recovery) report to the originating MISA OS W, which becomes aware of the recovery efforts being undertaken and awaits the outcome. The recovery is presented at the bottom right of Fig. 3, and consists of the release of the three connections and the establishment of a new one between the same boundary access points. It is now assumed that the connection can be established through the ATM subnetwork between QatmE1 and QatmE4. The alarm notification to the originating domain is part of the function Report Alarm from GBC Subnetwork Connection. This function informs the originating MISA OS W that the recovery of the affected connection is being undertaken by MISA OS E, so the originating MISA OS W takes no action.

The alarm report from domain $\mathrm{E}$ is sent to alarm correlator 1 in the originating MISA OS W together with that of the local alarm correlator 2 . The outcome of the correlation process identifies the failure in the context of the end-to-end connection.

Finally, the completion of the recovery procedure is reported, including the result, to the originating MISA OS W, making use of the Notification Recovery Process Result of the Xcoop interface. Both end users are in turn informed, completing the sequence of events.

\section{THE INFORMATION ARCHITECTURE OF THE XCOOP INTERFACE}

Going one step ahead toward the implementation of a system prototype, the functional requirements are mapped onto an information model. The above-mentioned managed resources are mapped to managed object classes (MOCs) and the management functions onto Common Management Information Protocol (CMIP) actions and notifications. During the specification procedure, the fundamental prerequisite has been the reuse of as many existing standard classes as possible. The result can be summarized in Fig. 4, which shows the inheritance tree.

In Fig. 4, the standard MOCs are distinguished by their labels, with references in parentheses. The NA4/X labels are from ETSI and the X.721 labels from the corre- 


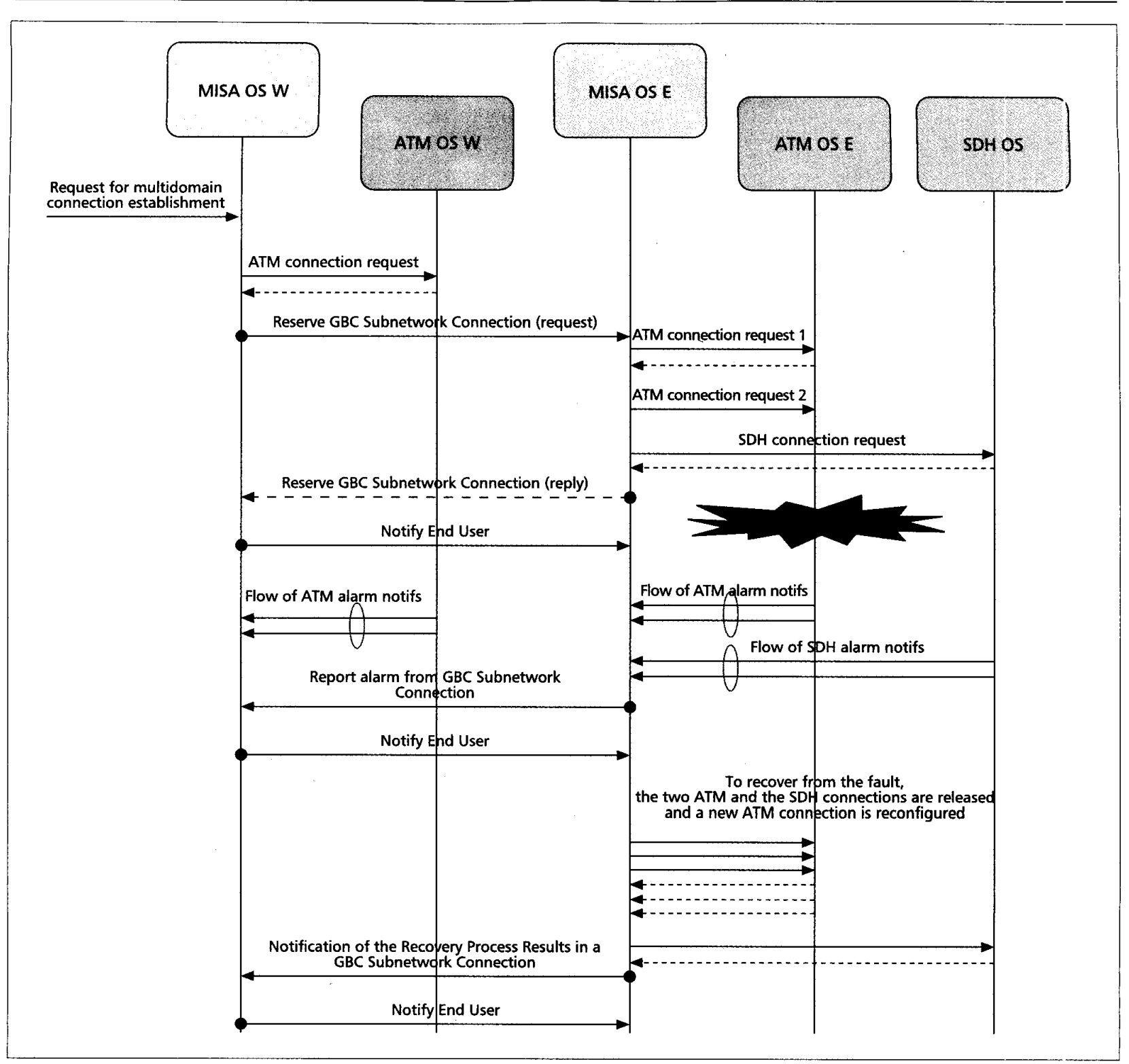

Figure 3. A time diagram of the management functions involved in a scenario considering reservation and fault recovery of a $G B C$ connection. Thicker arrows are Xcoop functions.

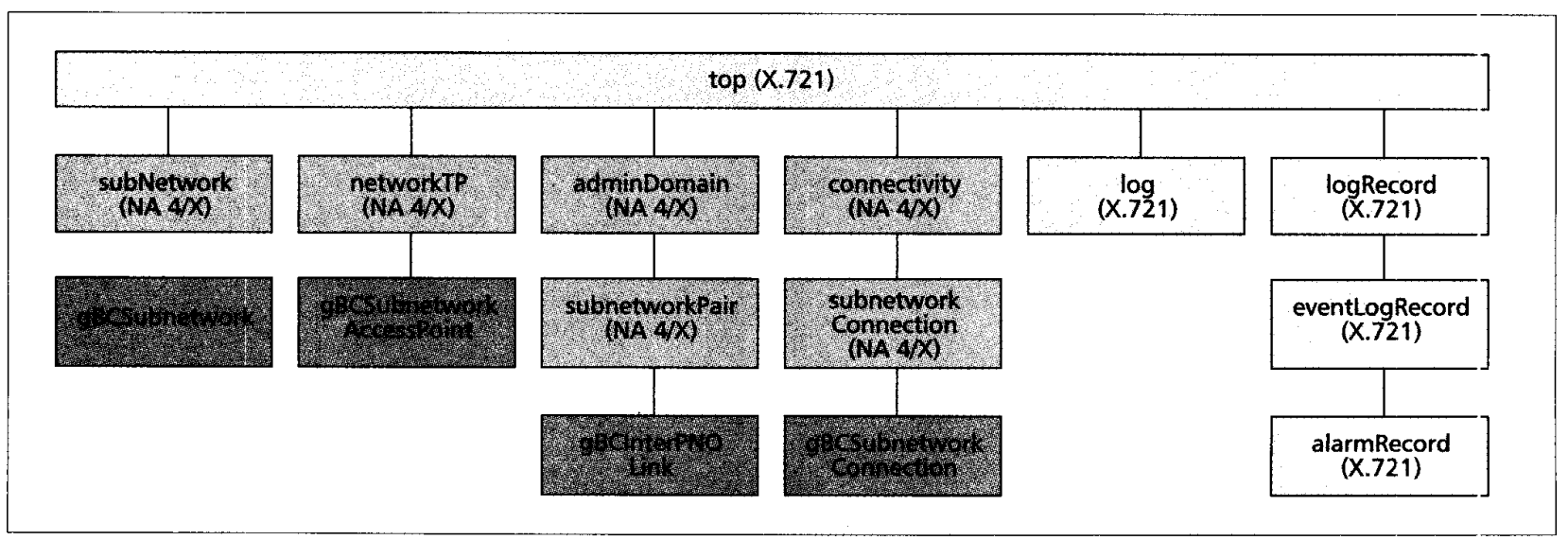

Figure 4. The inheritance tree of the Xcoop information model; NA4/X: ETSI standard; X.721: ITU-T Recommendation. 
sponding ITU-T Recommendation. On the bottom of the tree, the four Xcoop MOCs can be distinguished. It should be noted that although this model makes use of standard entities, it is the four additional defined classes that accomplish the needed functionality (i.e., the integration of ATM and SDH technologies). These specialized MISA MOCs meet the multitechnology requirements, defining both ATM and SDH attributes and characteristics and making them selectable by use of CHOICE clauses or OPTIONAL labels. A detailed description of the MOCs is beyond the scope of the article, but the reader is referred to the MISA specification documents [9] on this subject.

\section{ConClusions}

The Xcoop interface presented in this article meets the important requirements set by the need for seamless management services of multidomain networks, through covering the functional areas of Configuration and Fault Management. This Xcoop approach is unique in its competent management level, in that it integrates the management of two of the most promising and popular network technologies, ATM and SDH, successfully coping with such challenging topics as QoS, alarm reporting, recovery processes, and scheduling.

The implemented prototypes of this Xcoop model were tested in a pan-European trial environment especially set up to match real market situations and difficulties; the trial included four national hosts - Germany, Greece, Portugal, and Spain and illustrated excellent performance in terms of interoperability, compliance with specified behavior, and time measurements. The successful field trials strongly support the need for this Xcoop approach to handling raw bandwidth services in multidomain backbones operated by separate network operators.

For future evolution of the Xcoop model, the need to add highly efficient accounting management functionality has been identified, and the task is now approaching completion. Furthermore, integration of WDM management in the Xcoop model emerges as the next market demand. It is our belief and intention to extrapolate the concepts described in the present Xcoop model to include the new dense WDM technology in an integrated network management system, along with ATM and SDH technologies, consistent with the need and vision for seamless management of broadband networks.

\section{ACKNOWLEDGMENTS}

The authors wish to thank all other MISA partners for their invaluable contribution to the work reported in this article. The MISA project was cofunded by the European Union.

\section{REFERENCES}

[1] ITU-T Rec. M.3010, "Principles for a Telecommunications Management Network," Oct. 1992.

[2] EURESCOM P408, Del. 5/6, "Specification of the Xcoop Interface for SDH/ATM Network Management," Nov. 1996.

[3] ETSI EN 300 820-1 and -2, "Telecommunications Management Network (TMN); Management Information Model for the X-type interface between Operation Systems (OSs) of a Virtual Path (VP) / Virtual Channel (VC) cross-connected network," parts 1 and 2, July 1998.

[4] ETSI, EASI Project, European ATM Services Interoperability.

[5] TMF NMF 502, "Peer-to-Peer Service Configuration Business Agreement," issue 1.0, Apr. 1997.

[6] ACTS Project AC080. "Management of Integrated ATM and SDH Networks (MISA)," http://www.misa.ch/public

[7] D. Karali, et al., "QoS-based multi-domain routing in public broadband networks," Proc. 15\&N '98, Antwerp, Belgium, May 25-28, 1998.

[8] TMF NMF 025, "The Ensemble Concepts and Format," issue 1.0, Aug. 1992

[9] ACTS Project AC080, "Management of Integrated ATM and SDH Networks (MISA), Xcoop Interface, Ensembles and Information Model," Sept. 1998; http://www.misa.ch/public/ensembles.htm

[10] ETSI I-ETS-300653, "Telecommunications Management Network (TMN) Generic Managed Object Library for the Network Level View," May 1996.

\section{BIOGRAPHIES}

RAQUEL D IAZ-CALDERA (rdiaz@tsc.upc.es) graduated from the Universitat Politècnica de Catalunya (UPC), Spain in 1997 with the title of telecommunication engineer. She immediately joined the UPC team working on the European Union MISA project, where she contributed to the specification and develop ment of a TMN-Xcoop interface. She is currently working at IBM Research Laboratories, Zurich, Switzerland, and is focusing her doctoral thesis on mobile agent technology applications for service and network management.

JUAN SERRAT-FERNÁNDEZ (serrat@tsc.upc.es) graduated and received the title of doctor in telecommunication engineering from Universitat Politècnica de Catalunya, Spain, in 1977 and 1983, respectively. In 1986 he became associate professor with the Faculty of Telecommunication Engineering of UPC. He has being doing research in data communication networks and leading research projects funded by national entities and the European Union. His current interest is focused on provisioning of advanced communication services and integrated network management architectures and technologies.

KLEARCHOS BerDeKas (klearxos@telecom.ece.ntua.gr) received B.E. and M.Sc degrees in electrical and computer engineering from National Technical University of Athens (NTUA) in 1994 and Essex University in 1995, respectively, currently concluding his Ph.D. thesis at NTUA. Since 1995 he has been a research associate at the Telecommunications Laboratory, NTUA. He has conducted research in telecommunications network management including SNMP, TMN, and TINA/CORBA technologies for ISDN, ATM, SDH, WDM, and TCP/IP networks, in many EU and national projects.

FotIS KARAYANNIS (fkara@telecom.ntua.gr) graduated from the Greek-American Athens College in 1988 with honors. He received a D.Eng. degree from the Department of Electrical and Computer Engineering, NTUA, in 1994, and his $\mathrm{Ph} . \mathrm{D}$. in the field of management of integrated broadband multidomain networks in January 1999 from the same university. He has participated in several European Union co-funded projects like MONET, ICM, IDSM, and MISA. His current interest is in network and service management topics. 